\title{
KRAS G12C mutation as a poor prognostic marker of pemetrexed treatment in non-small cell lung
}

\section{cancer}

\author{
Sehhoon Park ${ }^{1, *}$, Ji-Yeon Kim ${ }^{1,}$, Se-Hoon Lee ${ }^{1}$, Beomseok Suh ${ }^{2}$, Bhumsuk Keam ${ }^{1}$, Tae Min Kim¹, \\ Dong-Wan Kim ${ }^{1}$, and Dae Seog $\mathrm{Heo}^{1}$
}

\begin{abstract}
${ }^{1}$ Department of Internal Medicine, ${ }^{2}$ Department of Family Medicine and Health Promotion Center, Seoul National University Hospital, Seoul, Korea
\end{abstract}

Received: September 1, 2015

Revised : October 12, 2015

Accepted: October 19, 2015

Correspondence to

Se-Hoon Lee, M.D.

Department of Internal Medicine, Seoul National University Hos-

pital, 101 Daehak-ro, Jongno-gu,

Seoul 03080, Korea

Tel: +82-2-2072-2199

Fax: +82-2-762-9662

E-mail: sehoon.lee119@gmail.com

*These authors contributed equally to this work.
Background/Aims: The predictive and prognostic value of KRAS mutation and its type of mutations in non-small cell lung cancer (NSCLC) are controversial. This clinical study was designed to investigate the predictive value of KRAS mutations and its mutation types to pemetrexed and gemcitabine based treatment. Methods: Advanced NSCLC patients tested for KRAS mutation ( $\mathrm{n}=334$ ) were retrospectively reviewed and 252 patients with wild type epidermal growth factor receptor and no anaplastic lymphoma kinase fusion were enrolled for the analysis. KRAS mutations were observed in 45 subjects with mutation type as followed: $\mathrm{G} 12 \mathrm{C}(\mathrm{n}=13), \mathrm{G} 12 \mathrm{D}(\mathrm{n}=12), \mathrm{G} 12 \mathrm{~V}(\mathrm{n}=12)$, other $(\mathrm{n}=8)$. Response rate $(\mathrm{RR})$, progression-free survival (PFS), and overall survival (OS) of pemetrexed singlet and gemcitabine based chemotherapy were analysis.

Results: Age, sex, performance status were well balanced between subjects with or without KRAS mutations. No difference was observed in RR. Hazard ratio (HR) of PFS for pemetrexed treated subjects with $\mathrm{G}_{12} \mathrm{C}$ mutation compared to subjects with KRAS wild type was 1.96 (95\% confidential interval [CI], 1.01 to $3.79 ; p=0.045$ ), but other mutations failed to show clinical significance. By analysis done by PFS, compared to the subjects with transition mutation, HR was 1.48 (95\% CI, 0.64 to 3.40; $p=0.360$ ) for subjects with transversion mutation on pemetrexed treatment and 0.41 (95\% CI, 0.19 to $0.87 ; p=0.020$ ) for subjects treated with gemcitabine based chemotherapy. No difference was observed in OS.

Conclusions: In this study, different drug sensitivity was observed according to the type of KRAS mutation. NSCLC subpopulations with different KRAS mutation type should be considered as different subgroups and optimal chemotherapy regimens should be searched in further confirmative studies.

Keywords: KRAS; Pemetrexed; Gemcitabine; Carcinoma, non-small-cell lung

\section{INTRODUCTION}

Non-small cell lung cancer (NSCLC), which constitutes $85 \%$ of lung cancer [1], is a leading cause of cancer related mortality [2]. However, prognosis of NSCLC differs based on its molecular profiles, and individualized therapy using targeted agents against activated signal pathways demonstrated promising prognosis $[3,4]$. Subpopulations without actionable molecular targets are still candidates of conventional cytotoxic chemotherapy and 
comparably poor prognosis is expected.

Approximately $54 \%$ of NSCLC patients have been identified with epidermal growth factor receptor (EGFR), KRAS mutations, or anaplastic lymphoma kinase (ALK) fusion usually mutually exclusive [5-7]. Among the mutations, KRAS mutations occur up to $30 \%$ of NSCLC patients [8], and higher mutation rates were observed in patients with previous history of smoking and adenocarcinoma histology type [9]. Former or current smokers were more likely to have transversion mutations $(\mathrm{G} \rightarrow \mathrm{T}$ or $\mathrm{G} \rightarrow \mathrm{C})$, in contrast to transition mutation $(\mathrm{G} \rightarrow \mathrm{A})$ being frequently observed in non-smokers [10]. More in detail, the majority of missense mutations occur primarily at codon 12 or 13, causing single amino acid substitutions at residues $\mathrm{G} 12$ or $\mathrm{G} 13$ [11].

The clinical implication of KRAS mutations as a predictive and prognostic marker is controversial. Some reports demonstrated increased overall survival (OS) hazard ratio (HR) in KRAS mutants compared with wild type [12-14], but others have failed to demonstrate consistent results $[15,16]$. Subgroup analysis done by location of amino acid substitution, codon 12 and codon 13, also failed to show significant difference in OS and response to chemotherapy [17]. As a predictive marker for response to chemotherapy, a recent preclinical study done by NSCLC cell line identified enhanced dependen-

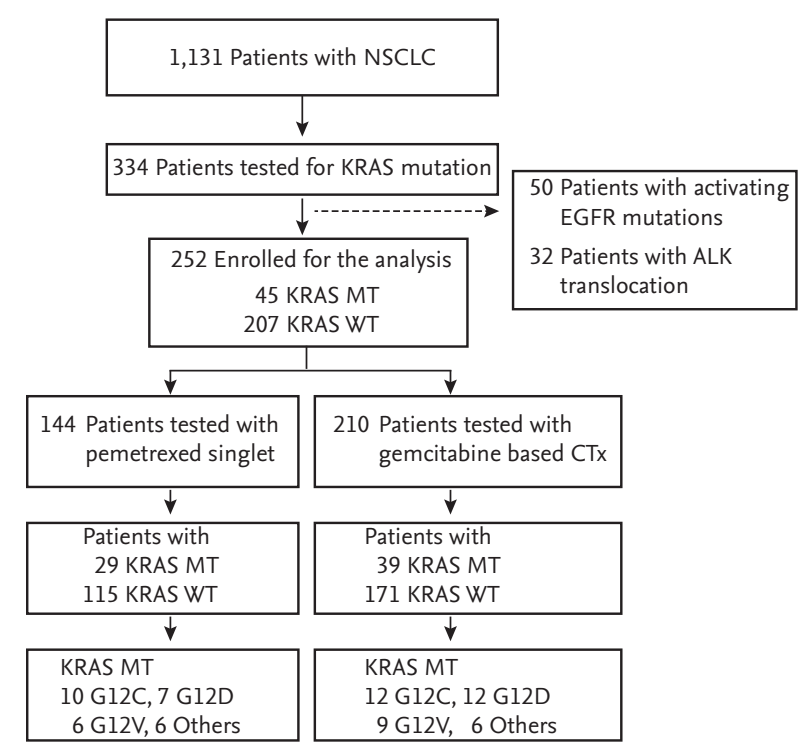

Figure 1. Flow chart of patients selection. NSCLC, nonsmall cell lung cancer; MT, mutation; WT, wild type; EGFR, epidermal growth factor receptor; ALK, anaplastic lymphoma kinase; CTx, cytotoxic chemotherapy. cy with anti-folate treatment by overexpression of genes within folate metabolism pathways in KRAS mutated cancer cells [18]. However, this result was also contrary to previous retrospective clinical studies [19,20].

Due to failure of small molecules targeting downstream of KRAS signal pathway [21,22], conventional cytotoxic chemotherapy is still recommended for NSCLC patients with KRAS mutations. Regarding the different KRAS epidemiology of each mutation among smokers and non-smokers, our study was designed to compare response of chemotherapy based on each type of KRAS mutation.

\section{METHODS}

\section{Study population}

NSCLC patients $(n=1,131)$ treated with conventional cytotoxic chemotherapy from July 2002 to December 2014 in Seoul National University Hospital (SNUH) were retrospectively reviewed. Out of 1,131 patients, 334 patients were tested for KRAS mutation status by direct DNA sequencing method. Test for KRAS mutation was conducted depends on availability of test and clinician's preference. Some patients were also tested for pre-screening purpose to decide eligibility of clinical trial enrollment. A total of 50 patients with activating EGFR mutations or unknown EGFR mutation status and 32 patients with ALK fusion were excluded. Analysis was conducted in two different groups. The first group consisted of 144 subjects treated with pemetrexed singlet chemotherapy and the second group consisted of 210 patients treated with gemcitabine based cytotoxic chemotherapy (Fig. 1). This study was approved by the SNUH Institutional Review Board (IRB No. 1412-103-634) and conducted in accordance with the World Medical Association's Declaration of Helsinki. Requirement for informed consent was waived.

\section{Data collection}

Each patient's medical record was retrospectively reviewed and medical history, pathology data, treatment history, imaging data, and genetic mutation results were acquired. Smokers were categorized as current former, or never smokers. Direct DNA sequencing method or peptide nucleic acid clamping test from tumor tissue was done to determine KRAS and EGFR mutation status. 
ALK translocation was tested by immunohistochemistry test. KRAS mutations were recorded more in detail by type of mutation, and categorized in four different groups: $\mathrm{G}_{12} \mathrm{C}, \mathrm{G} 12 \mathrm{D}, \mathrm{G} 12 \mathrm{~V}$, and mutations with other amino acid substitutions. Other mutations were Q22R, G13D, G12A, and G12F. Treatment response was evaluated by comparing post-treatment computed tomography (CT) to pre-treatment CT and in accordance to the Response Evaluation Criteria in Solid Tumor (RECIST) version 1.1. Survival data were collected from the Korean death registry and acquired from the Korean National Statistical Office.

The primary end point of our study was assessing the HR of each KRAS mutation by amino acid substitution to pemetrexed singlet treatment and gemcitabine based chemotherapy.

\section{Statistical analysis}

Baseline demographics of patients were analyzed with descriptive statistics between subjects with KRAS mutations and KRAS wild type. Additional analysis was conducted by subjects with four different categories by amino acid substitutions versus subjects with KRAS wild type, subjects with transition mutation and subjects with transversion mutation. Response rate by RECIST was compared with either chi-square test or Fisher exact test. HRs of progression-free survival (PFS) and OS were analyzed by Cox-proportional hazard regression analysis. Kaplan-Meier curves were used to portray the failure of treatment and calculate median PFS. Log-rank test was used to test the difference between the curves.

PFS of each patient was calculated from the date of initiation of palliative chemotherapy to the date of cancer progression or all-cause mortality. OS was calculated from the date of palliative setting of treatment to the date of cancer related mortality.

All results with two-sided $p$ value less than 0.05 were considered as significant and all the data were analyzed by STATA version 12.1 (StataCorp., College Station, TX, USA)

\section{RESULTS}

\section{Characteristics of study population}

Baseline clinical demographics of the total 252 patients are listed in Table 1. All subjects with KRAS mutations and KRAS wild type are well balanced. KRAS mutations were common in adenocarcinoma (86.7\%) as previously reported [9], but no statistical difference was observed by smoking status $(p=0.866)$. Eastern Cooperative Oncology Group (ECOG) performance status (PS) was categorized by subjects with ECOG PS o and 1 , and the subjects with poor performance status, ECOG PS 2, 3, and 4.

Approximately $95 \%$ of gemcitabine based chemotherapy was applied as first line chemotherapy. Pemetrexed singlet was applied as second line chemotherapy in 77.1\% of patients. Detailed description of subjects' profile by each type of KRAS mutations are shown in Table 1.

\section{Response rate of treatment}

Response rate of pemetrexed singlet treated patients $(\mathrm{n}=$ 144) between subjects with KRAS mutation and wild type failed to demonstrate significant difference $(p=0.464)$. Subgroup analysis done by the four categories showed no difference in response rate $(p=0.509)$.

All gemcitabine based chemotherapy regimens were combined $(n=210)$ and analyzed for difference in response rate. In the same manner, difference between KRAS mutation and wild type $(p=0.711)$, and difference between each type of KRAS mutation $(p=0.437)$ were insignificant (Table 2).

\section{Hazard ratio of progression-free survival and overall survival}

The relationship between KRAS mutations and PFS are portrayed by Kaplan-Meier curves in Fig. 2. PFS of KRAS mutant subjects treated with pemetrexed singlet ( $\mathrm{n}=$ 144) were compared to subjects with KRAS wild type and HR was calculated as 0.95 (95\% confidential interval [CI], 0.63 to $1.44 ; p=0.821)$. No difference in HR was observed in OS (HR, 0.95; 95\% CI, 0.58 to 1.56; $p=0.829$ ). Subgroup analysis by each amino acid substitution showed significantly shorter PFS in subjects with $\mathrm{G}_{12} \mathrm{C}$ mutation compared to KRAS wild type subjects (HR, 1.96; 95\% CI, 1.01 to $3.79 ; p=0.045)$. Other substitutions failed to show significant difference.

Same methods were applied to subjects treated with gemcitabine based chemotherapy $(n=210)$. Notably, subjects with transversion mutation showed decreased $\mathrm{HR}$ of PFS (HR, 0.41; 95\% CI, 0.19 to $0.87 ; p=0.020$ ) compared to subjects with transition mutation (Table 3, Fig. 3). No statistical significance was observed on survival analy- 
Table 1. Baseline characteristics of patients

\begin{tabular}{|c|c|c|c|c|c|c|c|}
\hline Characteristic & $\begin{array}{c}\text { KRAS WT } \\
(\mathrm{n}=207)\end{array}$ & $\begin{array}{c}\text { KRAS MT } \\
(\mathrm{n}=45)\end{array}$ & $p$ value & $\begin{array}{c}\mathrm{G} 12 \mathrm{C} \\
(\mathrm{n}=13)\end{array}$ & $\begin{array}{c}\mathrm{G} 12 \mathrm{D} \\
(\mathrm{n}=12)\end{array}$ & $\begin{array}{c}\mathrm{G} 12 \mathrm{~V} \\
(\mathrm{n}=12)\end{array}$ & $\begin{array}{c}\text { Others } \\
(\mathrm{n}=8)\end{array}$ \\
\hline Age, yr & $64(35-83)$ & $61(37-78)$ & $0.657^{\mathrm{a}}$ & $63(53-77)$ & $60(44-76)$ & $59(52-78)$ & $66(37-77)$ \\
\hline Sex & & & $0.752^{b}$ & & & & \\
\hline Male & $143(69.1)$ & $30(66.7)$ & & $12(92.3)$ & $4(33 \cdot 3)$ & $9(75.0)$ & $5(62.5)$ \\
\hline Female & $64(30.9)$ & $15(33 \cdot 3)$ & & $1(7.7)$ & $9(66.7)$ & $3(25.0)$ & $3(37 \cdot 3)$ \\
\hline Smoking & $126(60.9)$ & $28(62.2)$ & $0.866^{\mathrm{b}}$ & $12(92.3)$ & $3(25.0)$ & $9(75.0)$ & $4(50.0)$ \\
\hline Pathology & & & $0.024^{c}$ & & & & \\
\hline ADC & $153(74 \cdot 3)$ & $39(86.7)$ & & $11(84.6)$ & $11(91.7)$ & $11(91.7)$ & $6(75.0)$ \\
\hline BAC & $1(0.5)$ & $2(4.4)$ & & o & o & $1(8.3)$ & $1(12.5)$ \\
\hline SqCC & $17(8.3)$ & $1(2.2)$ & & o & $1(8.3)$ & o & o \\
\hline NOS & $35(16.7)$ & $3(6.7)$ & & $2(15 \cdot 4)$ & 0 & 0 & $1(12.5)$ \\
\hline ECOG PS & & & $0.829^{b}$ & & & & \\
\hline $\mathrm{o}$ and 1 & $164(79.2)$ & $35(77.8)$ & & $9(69.2)$ & $9(75.0)$ & $10(83.3)$ & $7(87.5)$ \\
\hline 2,3, and 4 & $43(20.8)$ & $10(22.2)$ & & $4(30.8)$ & $3(25.0)$ & $2(16.7)$ & $1(12.5)$ \\
\hline Recurrence after operation & $22(10.6)$ & $6(13 \cdot 3)$ & $0.601^{b}$ & $2(15 \cdot 4)$ & $2(16.7)$ & $2(16.7)$ & 0 \\
\hline Pemetrexed as & 115 & 29 & $0.696^{c}$ & & & & \\
\hline 1st line & $1(0.87)$ & 0 & & 0 & 0 & 0 & 0 \\
\hline 2nd line & $87(75 \cdot 7)$ & $24(82.8)$ & & $9(90.0)$ & $5(71.4)$ & $6(100.0)$ & $4(66.7)$ \\
\hline More than zrd line & $27(23.5)$ & $5(17.2)$ & & $1(10.0)$ & $2(28.6)$ & 0 & $2(33 \cdot 3)$ \\
\hline Gemcitabine based as & 171 & 39 & $1.000^{c}$ & & & & \\
\hline 1st line & $162(94.7)$ & $37(94.9)$ & & $12(100.0)$ & $10(83.3)$ & $9(100.0)$ & $6(100.0)$ \\
\hline 2nd line & $8(4 \cdot 7)$ & $2(5.1)$ & & o & $2(16.7)$ & 0 & o \\
\hline More than 3rd line & $1(0.6)$ & 0 & & 0 & 0 & 0 & o \\
\hline Gemcitabine based regimen & & & $0.985^{c}$ & & & & \\
\hline GP & $108(63.2)$ & $26(6.7)$ & & $7(58.3)$ & $8(66.7)$ & $7(77.8)$ & $4(66.6)$ \\
\hline $\mathrm{GC}$ & $44(25 \cdot 7)$ & $9(23.1)$ & & $5(41.7)$ & $2(16.7)$ & $1(11.1)$ & $1(16.7)$ \\
\hline Gemcitabine single & $11(6.4)$ & $2(5.1)$ & & 0 & $\mathrm{o}$ & $1(11.1)$ & $1(16.7)$ \\
\hline Other & $8(4.7)$ & $2(5.1)$ & & o & $2(16.7)$ & O & O \\
\hline
\end{tabular}

Values are presented as median (range) or number (\%).

WT, wild type; MT, mutation; ADC, adenocarcinoma; BAC, broncheoalveolar carcinoma; SqCC, squamous cell carcinoma; NOS, nitric oxide synthase; ECOG, Eastern Cooperative Oncology Group; PS, performance score; GP, gemcitabine and cisplatin; GC, gemcitabine and carboplatine.

${ }^{a} p$ value calculated by $t$ test.

b $p$ value calculated by chi-square test.

${ }^{c} p$ value calculated by Fisher exact test.

sis done by OS. More in detail, when subgroup analysis conducted with subjects treated with 1st line gemcitabine based chemotherapy ( $\mathrm{n}=199)$, transversion mutation showed HR 0.38 (95\% CI, 0.18 to $0.81 ; p=0.012$ ).

\section{DISCUSSION}

Despite the fact that KRAS mutations are common molecular changes in NSCLC, its utility as a clinical predictive marker was disappointing due to the controversial results [23]. Moreover, its role as a predictive marker of 
Table 2. Response rate to pemetrexed singlet treatment and gemcitabine based treatment by each type of KRAS mutation

\begin{tabular}{|c|c|c|c|c|c|c|}
\hline Value & CR & PR & $\mathrm{SD}$ & $\mathrm{PD}$ & NA & $p$ value $^{\mathrm{a}}$ \\
\hline \multicolumn{7}{|c|}{ Pemetrexed singlet treatment } \\
\hline KRAS WT & $1(0.9)$ & $27(23.8)$ & $35(30.4)$ & $52(45.2)$ & NA & $0.464^{b}$ \\
\hline KRAS MT & o & $10(34.5)$ & $10(34.5)$ & $9(31.0)$ & NA & \\
\hline $\mathrm{G} 12 \mathrm{C}$ & o & $3(30.0)$ & $2(20.0)$ & $5(50.0)$ & NA & $0.509^{c}$ \\
\hline $\mathrm{G} 12 \mathrm{D}$ & o & $3(42.9)$ & $2(28.6)$ & $2(28.6)$ & NA & \\
\hline $\mathrm{G} 12 \mathrm{~V}$ & o & $2(33 \cdot 3)$ & $4(66.7)$ & o & NA & \\
\hline Others & o & $2(33 \cdot 3)$ & $2(33 \cdot 3)$ & $2(33 \cdot 3)$ & NA & \\
\hline \multicolumn{7}{|c|}{ Gemcitabine based treatment } \\
\hline KRAS WT & $4(2.3)$ & $41(24.0)$ & $71(41.5)$ & $44(25 \cdot 73)$ & $11(6.4)$ & $0.711^{b}$ \\
\hline KRAS MT & $1(2.6)$ & $11(28.2)$ & $19(48.7)$ & $6(15 \cdot 4)$ & $2(5.1)$ & \\
\hline $\mathrm{G} 12 \mathrm{C}$ & o & $4(33 \cdot 3)$ & $8(66.7)$ & $\mathrm{O}$ & o & $0.437^{c}$ \\
\hline $\mathrm{G} 12 \mathrm{D}$ & ० & $2(16.7)$ & $5(41.7)$ & $4(33 \cdot 3)$ & $1(8.3)$ & \\
\hline $\mathrm{G} 12 \mathrm{~V}$ & $1(11.1)$ & $3(33 \cdot 3)$ & $3(33 \cdot 3)$ & $1(11.1)$ & $1(11.1)$ & \\
\hline Others & 0 & $2(33 \cdot 3)$ & $3(50.0)$ & $1(16.7)$ & 0 & \\
\hline
\end{tabular}

Values are presented as number (\%).

CR, complete response; PR, partial response; SD, stable disease; PD, progressive disease; NA, not available; WT, wild type; MT, mutation.

${ }^{a} p$ value calculated by Fisher exact test.

${ }^{\mathrm{b}} \mathrm{p}$ value between subjects with KRAS MT and KRAS WT.

${ }^{c} p$ value between each KRAS mutations.

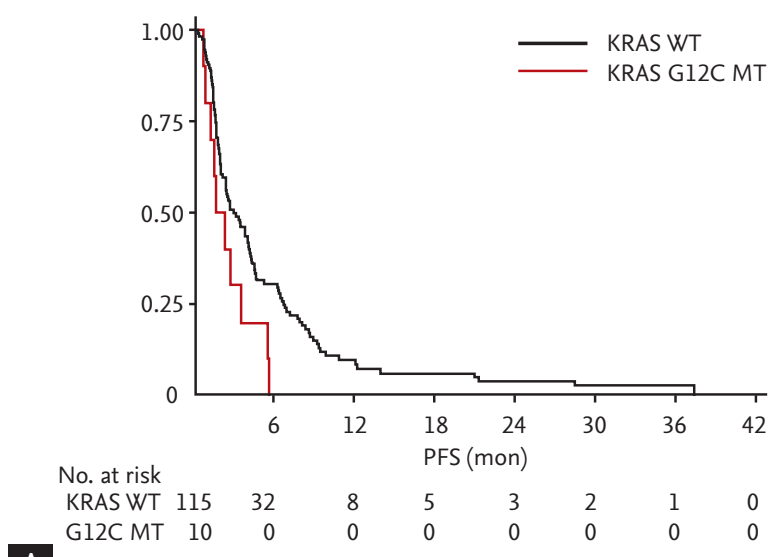

A

Figure 2. Kaplan-Meier curves of progression-free survival(PFS). PFS curve plotted by subjects treated by pemetrexed singlet. (A) Subjects with KRAS wild type (WT) and G12C mutations (MTs) ([median PFS, 2.9 months; 95\% confidential interval (CI), 2.3 to 4.0] vs. [median PFS, 1.6 months; $95 \%$ CI, 0.6 to 3.4], $p=0.040$ ); PFS curve plotted by subjects treated by gemcitabine based chemotherapy. (B) Subjects with KRAS wild type and G12C mutations ([median PFS, 4.8 months; $95 \%$ CI, 4.2 to 5.2] vs. [median PFS, 5.0 months; 95\% CI, 4.0 to 5.3], $p=0.761$ ).

cytotoxic chemotherapy had not been established. However, looking into more detail epidemiology of KRAS mutation, observation that KRAS transition mutation (G12D mutation) was more common in never smokers and transversion mutation ( $\mathrm{G} 12 \mathrm{C}, \mathrm{G} 12 \mathrm{~V}$ mutation) in smokers corresponded with the controversial results $[10,24,25]$. In a perspective view, shorter OS in NSCLC with smoking history were also observed in NSCLC pa- 
Table 3. Cox-proportional hazard ratios of pemetrexed singlet treatment and gemcitabine based treatment by each type of KRAS mutation

\begin{tabular}{|c|c|c|c|c|c|c|c|c|}
\hline \multirow{3}{*}{ Variable } & \multicolumn{4}{|c|}{ Pemetrexed singlet $^{\mathrm{a}}$} & \multicolumn{4}{|c|}{ Gemcitabine based treatment $^{b}$} \\
\hline & \multicolumn{2}{|l|}{ PFS } & \multicolumn{2}{|l|}{ OS } & \multicolumn{2}{|l|}{ PFS } & \multicolumn{2}{|l|}{ OS } \\
\hline & $\operatorname{HR}(95 \% \mathrm{CI})^{\mathrm{c}}$ & $p$ value $\mathrm{d}^{\mathrm{d}}$ & $\operatorname{HR}(95 \% \mathrm{CI})^{\mathrm{c}}$ & $p$ value $^{\mathrm{d}}$ & $\operatorname{HR}(95 \% \mathrm{CI})^{\mathrm{c}}$ & $p$ value $^{\mathrm{d}}$ & $\operatorname{HR}(95 \% \mathrm{CI})^{\mathrm{c}}$ & $p$ value $^{\mathrm{d}}$ \\
\hline KRAS MT & $0.95(0.63-1.44)$ & $0.821^{\mathrm{e}}$ & $0.95(0.58-1.56)$ & $0.829^{e}$ & $1.02(0.70-1.49)$ & $0.926^{\mathrm{e}}$ & $1.05(0.68-1.64)$ & $0.805^{\mathrm{e}}$ \\
\hline $\mathrm{G} 12 \mathrm{C}$ & $1.96(1.01-3.79)$ & $0.045^{\mathrm{e}}$ & $1.53(0.70-3.37)$ & $0.288^{\mathrm{e}}$ & $1.10(0.59-2.04)$ & $0.762^{\mathrm{e}}$ & $1.11(0.51-2.39)$ & $0.794^{\mathrm{e}}$ \\
\hline G12D & $0.70(0.32-1.51)$ & $0.359^{\mathrm{e}}$ & $1.31(0.57-3.05)$ & $0.525^{\mathrm{e}}$ & $1.57(0.82-3.01)$ & $0.171^{\mathrm{e}}$ & $1.15(0.58-2.27)$ & $0.696^{\mathrm{e}}$ \\
\hline $\mathrm{G} 12 \mathrm{~V}$ & $0.60(0.26-1.38)$ & $0.231^{e}$ & $0.39(0.12-1.26)$ & $0.115^{\mathrm{e}}$ & $0.55(0.24-1.24)$ & $0.148^{e}$ & $0.63(0.23-1.73)$ & $0.370^{e}$ \\
\hline Others & $1.09(0.48-2.49)$ & $0.838^{e}$ & $0.94(0.37-2.42)$ & $0.898^{\mathrm{e}}$ & $1.20(0.53-2.73)$ & $0.658^{e}$ & $1.56(0.63-3.84)$ & $0.335^{\mathrm{e}}$ \\
\hline Transversion MT & $1.48(0.64-3.40)$ & $0.360^{f}$ & $0.53(0.20-1.37)$ & $0.188^{f}$ & $0.41(0.19-0.87)$ & $0.020^{f}$ & $0.73(0.32-1.65)$ & $0.452^{f}$ \\
\hline Smoke & $1.16(0.82-1.65)$ & 0.400 & $1.14(0.73-1.78)$ & $0.560^{\mathrm{g}}$ & $0.90(0.66-1.22)$ & 0.508 & $1.04(0.72-1.49)$ & $0.849^{g}$ \\
\hline Chemotherapy line & $0.92(0.62-1.35)$ & 0.656 & $0.76(0.46-1.25)$ & $0.274^{h}$ & $1.50(0.79-2.86)$ & 0.217 & $0.97(0.53-1.78)$ & $0.925^{h}$ \\
\hline ECOG PS & $1.44(0.93-2.23)$ & 0.102 & $1.48(0.85-2.56)$ & $0.165^{\mathrm{i}}$ & $1.50(1.03-2.17)$ & 0.033 & $1.91(1.27-2.89)$ & $0.002^{i}$ \\
\hline
\end{tabular}

PFS, progression free survival; OS, overall survival; HR, hazard ratio; CI, confidence interval; MT, mutation; ECOG, Eastern Cooperative Oncology Group; PS, performance score.

${ }^{a}$ Analysis done with the subjects treated by pemetrexed singlet chemotherapy $(n=144)$.

${ }^{\mathrm{b}}$ Analysis done with the subjects treated by gemcitabine based chemotherapy $(\mathrm{n}=210)$.

${ }^{\mathrm{c}}$ Uni-variate analysis was done to calculate HR.

$\mathrm{d}$ p value calculated by cox-proportional analysis.

${ }^{\mathrm{e}}$ Compared to the subjects with KRAS wild type.

${ }^{\mathrm{f}}$ Compared to the subjects with transition mutation.

${ }^{\mathrm{g}}$ Compared to the subjects with never smoker.

${ }^{\mathrm{h}}$ Chemotherapy line considered as categorical variables, compared to the subjects treated with 1 st line.

${ }^{i}$ Compared to subjects with ECOG PS 2, 3, 4 to subjects with ECOG PS o, 1.

tients without considering mutational status [19]. Summarizing the above information, we have hypothesized and approached each type of KRAS mutation as a confounder of previous studies and as an independent variable in our study.

In this study, we have investigated the predictive value of each type of KRAS mutation to response from pemetrexed singlet treatment and gemcitabine based combination chemotherapy. As a result, subjects with G12C mutation are likely to demonstrate shorter PFS to pemetrexed treatment, and subjects with transition mutation (purine to pyrimidine or pyrimidine to purine mutation) are also expected to show shorter PFS to gemcitabine based treatment compared to wild type.

Similar result is evidenced in previous studies. The RASCAL (The Kirsten ras in-colorectal-cancer collaborative group) II study done with colon cancer patients showed that $\mathrm{G} 12 \mathrm{~V}$ mutation had poor prognosis in both PFS $(p<0.004)$ and OS $(p=0.008)$ [26]. In the BATTLE (The Biomarker-integrated Approaches of Targeted
Therapy for Lung Cancer Elimination) trial conducted with NSCLC patients, initial analysis done by overall presence of KRAS mutation failed to present clinical value for KRAS mutations [27], in which subgroup analysis comparing subjects with $\mathrm{G} 12 \mathrm{C}$ or G12V KRAS mutations to subjects with wild type or other type KRAS mutations showed shorter PFS $(p=0.046)$ [28]. Molecular structure modeling also confirmed that KRAS protein with $\mathrm{G} 12 \mathrm{C}$ changes are likely to activate the Ral (RAS-like) protein signaling pathway, unlike KRAS protein with G12D changes that are prone to activate RAS signaling cascade by activating phosphatidylinositol 3-kinase signaling pathways [28].

Different biology has been validated previously, but differences in drug sensitivity are unknown. A study conducted with NSCLC cell line show that G12C mutation is associated with a reduced response to cisplatin and increased response to pemetrexed. However, G12V mutation was sensitive in cisplatin and decreased in response to pemetrexed. No difference was observed 


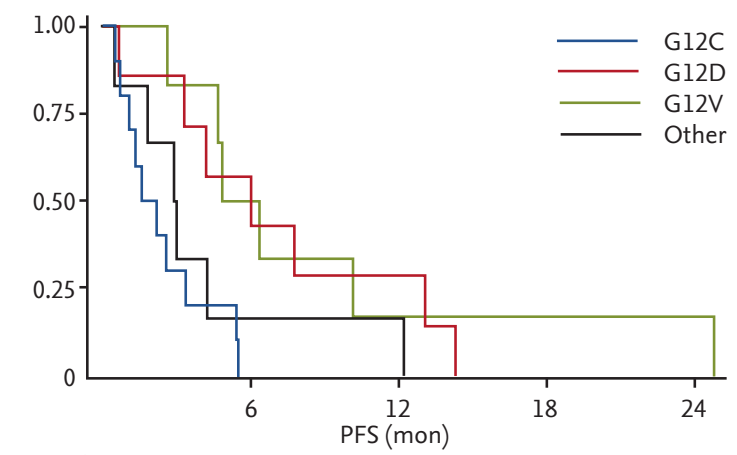

No. at risk

$\begin{array}{llllll}\text { G12C } 10 & 0 & 0 & 0 & 0 \\ \text { G12D } & 7 & 4 & 2 & 0 & 0 \\ \text { G12V } & 6 & 3 & 1 & 1 & 1 \\ \text { Other } & 6 & 1 & 1 & 0 & 0\end{array}$

A

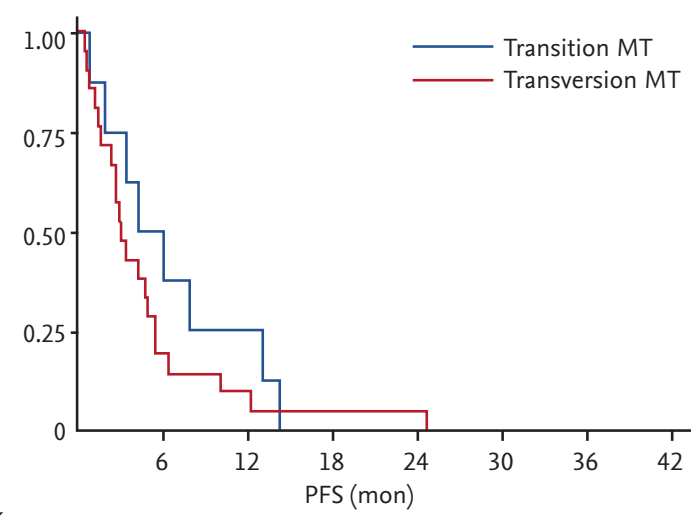

No. at risk

$\begin{array}{lrlllllll}\text { Transition MT } & 8 & 4 & 2 & 0 & 0 & 0 & 0 & 0\end{array}$

C

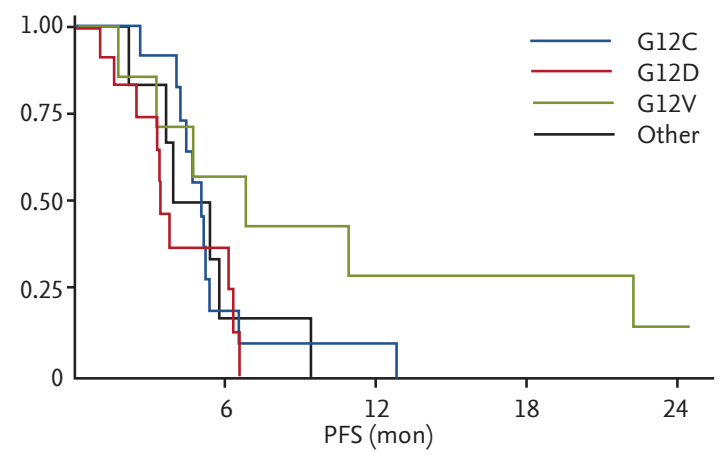

No. at risk

$\begin{array}{rrrrrr}\text { G12C } & 12 & 2 & 1 & 0 & 0 \\ \text { G12D } & 12 & 3 & 0 & 0 & 0 \\ \text { G12V } & 9 & 4 & 2 & 2 & 1 \\ \text { Other } & 6 & 1 & 0 & 0 & 0\end{array}$

B

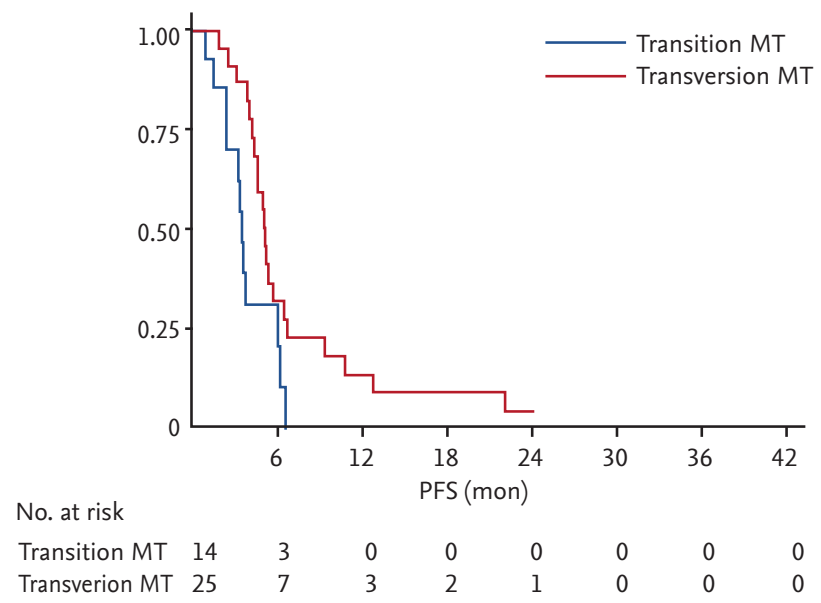

D

Figure 3. Kaplan-Meier curves of progression-free survival (PFS). (A, B) PFS curve plotted by each type of KRAS mutation (MT): (A) subjects with pemetrexed singlet treated; (B) subjects with gemcitabine based chemotherapy treated. (C, D) PFS curve plotted by transition mutation or transverse mutation: (C) subjects with pemetrexed singlet treatment; (D) subjects with gemcitabine based chemotherapy treatment.

between wild type and different KRAS mutations in response to gemcitabine [29]. But adverse results showing no correlation on folate receptor and KRAS were also reported [30]. In human studies, results were also controversial in retrospective studies comparing pemetrexed sensitivity by type of KRAS mutation. Although all studies have been reported in median OS, same tendency of poor respond to pemetrexed in subjects with $\mathrm{G}_{12} \mathrm{C}$ were demonstrated in a report [31] but another showed the opposite result [32].

Current guidelines for advanced NSCLC patients with- out activating mutations, including KRAS mutations, recommend starting treatment with pemetrexed, gemcitabine or taxane based treatment [33]. Due to limitation in our study, direct clinical application cannot be recommended, but it may be suggested that different treatment approach by type of KRAS mutation may be beneficial. G12C mutant subjects are likely to show early failure of pemetrexed treatment, and better response is expected in patients with $\mathrm{G} 12 \mathrm{D}$ and $\mathrm{G} 12 \mathrm{~V}$ mutations. In the same manner, subjects with transversion KRAS mutations are expected to better respond to gemcitabine 
based treatment compared to subjects with transition mutations (Table 3). Statistical significance was not satisfied for all the categories and direct comparison between regimens were not allowed, nonetheless, different tendency in response were observed by each mutation.

Our study has limitations. Subjects with KRAS mutations were limited in number as well as heterogeneous chemotherapy regimens were applied. Hence, statistical power for subgroup analysis was limited. Further pooled analysis should be conducted by each type of KRAS mutation to validate our results. Currently, pemetrexed singlet treatment is not routinely used for NSCLC patients. However due to the national insurance reimbursement policy in South Korea, we were allowed to analyze subjects treated with singlet pemetrexed which is meaningful because direct clinical effect of pemetrexed by each mutation without other confounding drug effect could be considered. Although our results have limitation to suggest clear clinical implication for patients with KRAS mutations, to our knowledge, this study is the first clinical study to test drug sensitivities by each mutation.

In conclusion, we have demonstrated G12C KRAS mutation showed reduced PFS to pemetrexed singlet treatment and value of transversion KRAS mutation as a good predictive marker of gemcitabine based chemotherapy. Further studies are warranted for confirmation.

\section{KEY MESSAGE}

1. Non-small cell lung cancer subpopulations with different KRAS mutation showed different sensitivity to drug.

2. In patients with G12C KRAS mutation showed reduced response to pemetrexed treatment.

3. Transversion mutation showed good response to gemcitabine based chemotherapy.

\section{Conflict of interest}

No potential conflict of interest relevant to this article was reported.

\section{Acknowledgments}

This study was supported by a grant from the Innovative Research Institute for Cell Therapy, Republic of Korea (Ao62260).

\section{REFERENCES}

1. Molina JR, Yang P, Cassivi SD, Schild SE, Adjei AA. Nonsmall cell lung cancer: epidemiology, risk factors, treatment, and survivorship. Mayo Clin Proc 2008;83:584-594.

2. Jemal A, Bray F, Center MM, Ferlay J, Ward E, Forman D. Global cancer statistics. CA Cancer J Clin 2011;61:69-90.

3. Rosell R, Carcereny E, Gervais R, et al. Erlotinib versus standard chemotherapy as first-line treatment for European patients with advanced EGFR mutation-positive non-small-cell lung cancer (EURTAC): a multicentre, open-label, randomised phase 3 trial. Lancet Oncol 2012;13:239-246.

4. Solomon BJ, Mok T, Kim DW, et al. First-line crizotinib versus chemotherapy in ALK-positive lung cancer. N Engl J Med 2014;371:2167-2177.

5. Suda K, Tomizawa K, Mitsudomi T. Biological and clinical significance of KRAS mutations in lung cancer: an oncogenic driver that contrasts with EGFR mutation. Cancer Metastasis Rev 2010;29:49-60.

6. Shankaran V, Obel J, Benson AB 3rd. Predicting response to EGFR inhibitors in metastatic colorectal cancer: current practice and future directions. Oncologist 2010;15:157-167.

7. Kris MG, Johnson BE, Kwiatkowski DJ, et al. Identification of driver mutations in tumor specimens from 1,000 patients with lung adenocarcinoma: the NCI's Lung Cancer Mutation Consortium (LCMC). J Clin Oncol 2011;29:CRA7506.

8. Graziano SL, Gamble GP, Newman NB, et al. Prognostic significance of K-ras codon 12 mutations in patients with resected stage I and II non-small-cell lung cancer. J Clin Oncol 1999;17:668-675.

9. Mao C, Qiu LX, Liao RY, et al. KRAS mutations and resistance to EGFR-TKIs treatment in patients with nonsmall cell lung cancer: a meta-analysis of 22 studies. Lung Cancer 2010;69:272-278.

10. Riely GJ, Kris MG, Rosenbaum D, et al. Frequency and distinctive spectrum of KRAS mutations in never smokers with lung adenocarcinoma. Clin Cancer Res 2008;14:5731-5734.

11. Schubbert S, Shannon K, Bollag G. Hyperactive Ras in developmental disorders and cancer. Nat Rev Cancer 2007;7:295-308.

12. Mascaux C, Iannino N, Martin B, et al. The role of RAS oncogene in survival of patients with lung cancer: a sys- 
tematic review of the literature with meta-analysis. Br J Cancer 2005;92:131-139.

13. Cadranel J, Mauguen A, Faller M, et al. Impact of systematic EGFR and KRAS mutation evaluation on progression-free survival and overall survival in patients with advanced non-small-cell lung cancer treated by erlotinib in a French prospective cohort (ERMETIC project: part 2). J Thorac Oncol 2012;7:1490-1502.

14. Meng D, Yuan M, Li X, et al. Prognostic value of K-RAS mutations in patients with non-small cell lung cancer: a systematic review with meta-analysis. Lung Cancer 2013;81:1-10.

15. Zalcman G, Beau-Faller M, Creveuil C, et al. Use of Ras effector RASSF1A promoter gene methylation and chromosome op loss of heterozygosity ( $\mathrm{LOH}$ ) to predict progression-free survival (PFS) in perioperative chemotherapy (CT) phase III trial IFCT-0oo2 in resectable non-small cell lung cancer. J Clin Oncol 2008;26(15 Suppl):7500.

16. D'Angelo SP, Janjigian YY, Ahye N, et al. Distinct clinical course of EGFR-mutant resected lung cancers: results of testing of 1118 surgical specimens and effects of adjuvant gefitinib and erlotinib. J Thorac Oncol 2012;7:1815-1822.

17. Loriot Y, Mordant P, Deutsch E, Olaussen KA, Soria JC. Are RAS mutations predictive markers of resistance to standard chemotherapy? Nat Rev Clin Oncol 2009;6:528534 .

18. Moran DM, Trusk PB, Pry K, Paz K, Sidransky D, Bacus SS. KRAS mutation status is associated with enhanced dependency on folate metabolism pathways in non-small cell lung cancer cells. Mol Cancer Ther 2014;13:1611-1624.

19. Sun JM, Hwang DW, Ahn JS, Ahn MJ, Park K. Prognostic and predictive value of KRAS mutations in advanced non-small cell lung cancer. PLoS One 2013;8:e64816.

20. Rodenhuis S, Boerrigter L, Top B, et al. Mutational activation of the K-ras oncogene and the effect of chemotherapy in advanced adenocarcinoma of the lung: a prospective study. J Clin Oncol 1997;15:285-291.

21. Janne PA, Shaw AT, Pereira JR, et al. Phase II double-blind, randomized study of selumetinib (SEL) plus docetaxel (DOC) versus DOC plus placebo as second-line treatment for advanced KRAS mutant non-small cell lung cancer (NSCLC). J Clin Oncol 2012;30:7503.

22. Sequist LV, von Pawel J, Garmey EG, et al. Randomized phase II study of erlotinib plus tivantinib versus erlotinib plus placebo in previously treated non-small-cell lung cancer. J Clin Oncol 2011;29:3307-3315.

23. Roberts PJ, Stinchcombe TE. KRAS mutation: should we test for it, and does it matter? J Clin Oncol 2013;31:11121121.

24. Lee YJ, Kim JH, Kim SK, et al. Lung cancer in never smokers: change of a mindset in the molecular era. Lung Cancer 2011;72:9-15.

25. Dogan S, Shen R, Ang DC, et al. Molecular epidemiology of EGFR and KRAS mutations in 3,026 lung adenocarcinomas: higher susceptibility of women to smoking-related KRAS-mutant cancers. Clin Cancer Res 2012;18:61696177.

26. Andreyev HJ, Norman AR, Cunningham D, et al. Kirsten ras mutations in patients with colorectal cancer: the 'RASCAL II' study. Br J Cancer 2001;85:692-696.

27. Kim ES, Herbst RS, Wistuba II, et al. The BATTLE trial: personalizing therapy for lung cancer. Cancer Discov 2011;1:44-53.

28. Ihle NT, Byers LA, Kim ES, et al. Effect of KRAS oncogene substitutions on protein behavior: implications for signaling and clinical outcome. J Natl Cancer Inst 2012;104:228-239.

29. Garassino MC, Marabese M, Rusconi P, et al. Different types of K-Ras mutations could affect drug sensitivity and tumour behaviour in non-small-cell lung cancer. Ann Oncol 2011;22:235-237.

30. Nunez MI, Behrens C, Woods DM, et al. High expression of folate receptor alpha in lung cancer correlates with adenocarcinoma histology and EGFR [corrected] mutation. J Thorac Oncol 2012;7:833-840.

31. Macerelli M, Caramella C, Faivre L, et al. Does KRAS mutational status predict chemoresistance in advanced nonsmall cell lung cancer (NSCLC)? Lung Cancer 2014;83:383388.

32. Kamdar MK, Parent T, Walker PR. KRAS gene mutation status and sensitivity to pemetrexed: a retrospective analysis. J Clin Oncol 2013;31:e19154.

33. Reck M, Popat S, Reinmuth N, et al. Metastatic nonsmall-cell lung cancer (NSCLC): ESMO Clinical Practice Guidelines for diagnosis, treatment and follow-up. Ann Oncol 2014;25 Suppl 3:iii27-iiiz9. 